\title{
Horas académico-asistenciales en el nuevo Reglamento del Residentado Médico en Perú
}

\author{
Academic and work hours in the new Regulation of the Medical Residency in Peru
}

\section{Sr. Editor:}

En marzo del 2017 se aprobó el Reglamento de la Ley del Sistema Nacional de Residentado Médico (SINAREME) (1); este reglamento tiene como objeto regular el funcionamiento y desarrollo del Sistema Nacional de Residentado Médico. Tras revisar su contenido, consideramos importante evaluar el artículo 36 que detalla las obligaciones del médico residente, con énfasis en lo pertinente a las horas dedicadas.

El primer inciso establece que el residente deberá cumplir como mínimo 60 horas semanales en actividades académico - asistenciales, mas no determina un número máximo de horas, quedando incierto el tiempo máximo de permanencia. Lo contrario sucede con el programa de residentado de los Estados Unidos que fija como máximo 80 horas semanales; basándose en evidencias, en marzo del presente año se aprobó una mayor flexibilización para el cumplimiento de dichas horas (2); por ejemplo, el ensayo FIRST (que incluyó a 119 programas de residencia en cirugía) reveló que al incorporar las reglas de flexibilización horaria los resultados no fueron inferiores en relación a la seguridad del paciente (3). En tanto, se esperan los resultados del ensayo iCOMPARE que incluye 63 programas de residencia médica y que también explora la flexibilidad horaria. Si bien en el Perú, la media de horas de trabajo a la semana cumplidas por los residentes oscilaría entre 80 a 90 horas (4), debería señalarse un número máximo de horas para lograr un mayor orden en la distribución de actividades.

De otro lado, el inciso 4 menciona que el residente deberá permanecer hasta las 13:00 horas tras concluir su guardia nocturna para hacer efectivo su descanso post-guardia. Es conocido que en la mayoría de casos el residente ingresa a la guardia programada luego de cumplir su turno regular en servicio. Es decir, no tiene descanso previo a la guardia; además, no tiene asignado un periodo corto de descanso durante la misma y tampoco tras concluirla. La privación del sueño conduce a alteraciones en el desempeño, los procesos cognitivos, el ánimo y bienestar del residente, además de poner en riesgo la seguridad del paciente (5). Por más que en las sedes hospitalarias se tomen en cuenta estas importantes consecuencias y decidan aplicar el descanso inmediato post-guardia, se verían imposibilitadas por lo normado en el reglamento.

Por último, el inciso 5 indica que el residente tiene derecho a un descanso continuo de 24 horas a la semana; si bien es un importante beneficio para el profesional, su ausencia provocaría algunas desventajas. En el ámbito académico, podría perder alguna exposición importante. En cuanto a la actividad asistencial, tendría un desfase respecto a la evolución de sus pacientes hospitalizados e información sobre los nuevos ingresos. Siendo así, sería más conveniente la aplicación de la flexibilidad horaria que permita al residente establecer su tiempo de permanencia y repartir dichas horas de descanso según su propio criterio.

\section{Carlos Orellano ${ }^{1,2, a, b}$, Joshep Revilla ${ }^{1,3, b}$}

\section{Correspondencia:}

Joshep Revilla.

Av. Honorio Delgado 430. San Martín de Porres. Lima - Perú.

Correo electrónico: joshep.revilla.z@upch.pe

\footnotetext{
Facultad de Medicina Alberto Hurtado, Universidad Peruana Cayetano Heredia. Lima, Perú.

Hospital Cayetano Heredia. Lima, Perú.

Instituto Nacional de Salud Mental Honorio Delgado - Hideyo Noguchi. Lima, Perú.

Magister en Informática Biomédica en Salud Global;

Médico residente de psiquiatría
} 


\section{REFERENCIAS BIBLIOGRÁFICAS}

1. Congreso de la República del Perú. Decreto Supremo $\mathrm{N}^{\circ}$ 007-2017-SA. Reglamento de la Ley N ${ }^{\circ} 30453$, Ley del Sistema Nacional de Residentado Médico (SINAREME). Lima: Diario Oficial El Peruano; 01 de marzo del 2017.

2. Asch DA, Bilimoria KY, Desai SV. Resident Duty Hours and Medical Education Policy - Raising the

Evidence Bar. N Engl J Med. 2017; 376(18):1704-6.

3. Bilimoria KY, Chung JW, Hedges LV, et al. National cluster-randomized trial of duty-hour flexibility in surgical training. N Engl J Med. 2016; 374(8):71327.
4. Mariños A, Otero M, Málaga G, Tomateo D. Coexistencia de síndrome de Burnout y síntomas depresivos en médicos residentes. Estudio descriptivo transversal en un hospital nacional de Lima. Rev Med Hered. 2011; 22(4):159-60.

5. Mansukhani MP, Kolla BP, Surani S, Varon J, Ramar K. Sleep deprivation in resident physicians, work hour limitations, and related outcomes: a systematic review of the literature. Postgrad Med. 2012; 124(4):241-9.

Recibido: 11/08/2017 